\title{
RANDOM WALKS ON COMPACT SEMIGROUPS
}

\author{
A. MUKHERJEA, T. C. SUN AND N. A. TSERPES
}

ABstract. Let $\beta$ be a regular Borel probability measure with support $F$ on a compact semigroup $S$. Let $X_{1}, X_{2}, \cdots$ be a sequence of independent random variables on some probability space $(\Omega, \Sigma, P)$ with values in $S$, having identical distribution $P\left(X_{n} \in B\right)=$ $\beta(B)$. The random walk $Z_{n}=X_{1} X_{2} \cdots X_{n}$ is studied in this paper. Let $D$ be the closed semigroup generated by $F$. An element $x$ in $D$ is called recurrent iff $P_{x}\left(Z_{n} \in N_{x}\right.$ i.o. $)=1$ for every open set $N_{x}$ containing $x$. This paper characterizes the recurrence of an element $x$ in terms of divergence of the series $\sum_{n=1}^{\infty} \beta^{n}\left(N_{x}\right)$ for every open set $N_{x}$ containing $x$. It also shows that the set of recurrent states of $\left\{Z_{n}\right\}$ is precisely the kernel of $D$.

1. Let $S$ be a compact semigroup, i.e. a compact Hausdorff space which is algebraically a semigroup with jointly continuous multiplication. Let $\beta$ be a regular probability measure defined on the Borel subsets of $S$. Let $X_{1}, X_{2}, \cdots$ be a sequence of independent random variables on some probability space $(\Omega, \Sigma, P)$ with values in $S$, having identical distribution $P\left(X_{n} \in B\right)=\beta(B)$ for Borel sets $B$ in $S$. For probabilistic considerations, we will, with no loss of generality, identify the process $X_{n}$ with the usual coordinate representation process in the sequence space $S^{\infty}$. The sequence $Z_{n}=X_{1} X_{2} \cdots X_{n}, n=1,2, \cdots$, is called a right random walk on $S$ generated by $\beta$. The sequence $W_{n}=X_{n} X_{n-1} \cdots X_{1}, n=1,2, \cdots$, is analogously called a left random walk on $S$. The set $D=\operatorname{cl}\left(\bigcup_{n=1}^{\infty} F^{n}\right)$, the closed subsemigroup generated by $F=\{x \in S$ : every open set containing $x$ has positive $\beta$-measure $\}=$ the support of $\beta$, is called the support of the random walk $\left\{Z_{n}\right\}$ or $\left\{W_{n}\right\}$. By the kernel $K$ of $D$, we mean the minimal two-sided ideal (which always exists since $D$ is a compact semigroup) of $D$. It is well known that $K$ is a completely simple semigroup (i.e. simple and contains a primitive idempotent) and, for every $x \in K, x K=x D$ is a minimal right ideal of $D$ and contains an idempotent. For these facts, we refer to [1].

Throughout this paper, we will use the following notations.

$$
\begin{aligned}
y^{-1} B & =\{x \in S: y x \in B\}, \\
A^{-1} B & =\bigcup\left\{y^{-1} B: y \in A\right\} \quad \text { and } \\
Z_{k}^{-1} Z_{n} & =X_{k+1} X_{k+2} \cdots X_{n} .
\end{aligned}
$$

Received by the editors June 19, 1972 and, in revised form, December 18, 1972.

AMS (MOS) subject classifications (1970). Primary 60G50, 60J15, 43A05.

Key words and phrases. Topological semigroup, kernel of a semigroup, recurrent random walks. 
Let $\beta^{n}$ denote $\beta^{*} \beta^{*} \cdots * \beta$ ( $n$-times). Then $P\left(Z_{k} \in B\right)=\beta^{k}(B)$ and $P\left(Z_{n+k} \in B \mid Z_{n}=x\right)=\beta^{k}\left(x^{-1} B\right)$. [Note that implicitly we have assumed that the $Z_{n}$ 's are measurable in the sequence probability space. The measurability of the $Z_{n}$ 's is, of course, a fact when $S$ is second countable.]

An element $x \in D$ is called a recurrent state for $\left\{Z_{n}\right\}$ if

$$
P_{x}\left(Z_{n} \in N_{x} \text { i.o. }\right) \equiv P\left(Z_{n} \in N_{x} \text { infinitely often } \mid Z_{1}=x\right)=1
$$

for every open set $N_{x}$ containing $x$. A random walk having at least one recurrent state is called recurrent. Recurrent random walks were studied recently by Rosenblatt [8], Martin-Löff [6] and Larisse ([3], [4]) in the case of discrete semigroups and by the authors [10] in the case of compact abelian and completely simple semigroups. In [10], the authors showed that the set of recurrent states of the random walk $\left\{Z_{n}\right\}$ on a compact abelian $S$ is precisely the kernel $K$ of $D$. The methods in [10] exploited the abelian property of $S$ and so could not be extended to the nonabelian case.

The purpose of this paper is to characterize the recurrence of an element $x \in D$ in terms of divergence of the series $\sum_{n=1}^{\infty} \beta^{n}\left(N_{x}\right)$ for every open set $N_{x}$ containing $x$ and to show that the set of recurrent states of $\left\{Z_{n}\right\}$ is precisely the kernel $K$ of $D$, in the case of compact semigroups $S$.

It is relevant to mention that the same problem of recurrence was first considered by Chung and Fuchs on the Euclidean $n$-space in [2] and by Loynes on locally compact and compact groups in [5] with a little different definition which is equivalent to our definition on groups. Our definition of recurrence on semigroups differs from that of Chung and Fuchs or Loynes since one can easily find compact semigroups where there are random walks which are not recurrent in their sense; for instance, if $S=E \times G$, where $G=\{1, a\}$ is a group and $E=\left\{e_{1}, e_{2}\right\}$ is a left-zero semigroup and $\beta$ is the normed counting measure on $S$, then it is easy to see that, for every $x$ in $S, P\left(Z_{n}=x\right.$ i.o. $)=\frac{1}{2}<1$.

In what follows, $S$ (and hence $D$ ) is always compact. We write: (a) $x \rightarrow y$ when $y \in x D$ and $x, y$ elements of $D$ (b) $x \rightarrow y$ i.o. when

$$
P_{x}\left(Z_{n} \in N_{y} \text { i.o. }\right)=1
$$

for every open set $N_{y}$ containing $y$, for $x, y$ elements of $D$.

2. Before we present our main results, we need the following lemmas.

Lemma 2.1. Given $x \in S$ and $N_{y}$, an open set containing $y$ in $S$, we can find open sets $N_{1}$ and $N_{2}$ containing $x$ and $y$ respectively such that $N_{1}^{-1} N_{2} \subset$ $x^{-1} N_{y}$. 
Proof. Suppose first that $x^{-1} N_{y}$ is empty. Then $y \notin x S$. If for every open set $N^{\prime}$ containing $y$ and open set $N^{\prime \prime}$ containing $x, N^{\prime} \cap N^{\prime \prime} S$ is nonempty, then we can find a net $y_{u}$ converging to $y$ and a net $x_{u}$ converging to $x$ such that $y_{u}=x_{u} s_{u}$, where $s_{u}$ 's are elements in $S$. Since $S$ is compact, we can find a subnet of $s_{u}$ converging to some element $s$ in $S$. But then $y=x s$ and this contradicts that $x^{-1} N_{y}$ is empty. Hence, when $x^{-1} N_{y}$ is empty, there exist open sets $N_{1}$ and $N_{2}$ containing $x$ and $y$ respectively such that $N_{2} \cap N_{1} S$ is empty which means that $N_{1}^{-1} N_{2}$ is empty.

Suppose next that $x^{-1} N_{y}$ is nonempty. Suppose, for every open set $N^{\prime}$ containing $y$ and contained in $N_{0}$, a compact neighbourhood of $y$ such that $N_{0} \subset N_{y}$, and every open set $N^{\prime \prime}$ containing $x$, we have $N^{\prime \prime-1} N^{\prime} \cap$ $\left(x^{-1} N_{y}\right)^{c}$ is nonempty. Then we can find a net $x_{u}$ converging to $x$ and $z_{u} \in N^{\prime \prime-1} N^{\prime} \cap\left(x^{-1} N_{y}\right)^{c}$ such that $x_{u} z_{u} \in N_{0}$ and $x z_{u} \notin N_{y}$. Since $S$ is compact, we can find a subnet of $z_{u}$ converging to $z$ such that $x z \in N_{0}$ and $x z \notin N_{y}$. This is a contradiction and the lemma follows.

Lemma 2.2. If $x \rightarrow y$ i.o. and $x \rightarrow z$, then $z \rightarrow y$ i.o.

Proof. If $z+y$ i.o., then there is an open set $N$ containing $y$ such that $P_{z}\left(Z_{n} \in N\right.$ finitely often $)>0$. By Lemma 2.1 , we can find open sets $N_{z}$ and $N_{y}$ containing $z$ and $y$ respectively such that $N_{z}^{-1} N_{y} \subset z^{-1} N$. But then $P\left(Z_{n} \in N_{z}^{-1} N_{y}\right.$ finitely often $)>0$ and

(1) $P_{x}\left(Z_{n} \in N_{y}\right.$ finitely often $) \geqq P_{x}\left(Z_{k} \in N_{z}, Z_{n} \in N_{y}\right.$ finitely often $)$

$\geqq P_{x}\left(Z_{k} \in N_{z}, Z_{k}^{-1} Z_{n} \in N_{z}^{-1} N_{y}\right.$ finitely often)

$=P_{x}\left(Z_{k} \in N_{z}\right) P\left(Z_{n} \in N_{z}^{-1} N_{y}\right.$ finitely often $)$

$\geqq P_{x}\left(Z_{k} \in N_{z}\right) P\left(Z_{n} \in z^{-1} N\right.$ f.o. $)>0$,

if $Z_{k}$ is such that $P_{x}\left(Z_{k} \in N_{z}\right)>0$. This is a contradiction to the fact that $x \rightarrow y$ i.o. and the lemma follows.

Lemma 2.3. The set $R$ of the recurrent states of $\left\{Z_{n}\right\}$ is a subset of the kernel $K$ of $D$.

Proof. Let $x \in R$. Then $x \in x D$. We claim that $x D$ is a minimal right ideal of $D$. To prove this, let $a \in x D$. Suppose that $x \notin a D$. Then, by Lemma 2.1 , we can find open sets $N_{x}$ and $N_{a}$ containing $x$ and $a$ respectively such that $N_{a}^{-1} N_{x} \cap D$ is empty. Since $a \in x D$, for some positive integer $k, P_{x}\left(Z_{k} \in N_{a}\right)>0$. Now

$$
\begin{aligned}
P_{x}\left(Z_{n} \in N_{x} \text { finitely often }\right) & \geqq P_{x}\left(Z_{k} \in N_{a}, Z_{n+k} \in N_{x} \text { finitely often }\right) \\
& \geqq P_{x}\left(Z_{k} \in N_{a}, Z_{k}^{-1} Z_{n+k} \in N_{a}^{-1} N_{x} \text { finitely often }\right) \\
& =P_{x}\left(Z_{k} \in N_{a}\right)>0 .
\end{aligned}
$$


This contradicts that $x \in R$ and therefore, $x \in a D$ so that $x D$ is a minimal right ideal of $D$. Since the kernel $K$ of $D$ is the union of all minimal right ideals of $D, x \in K$.

THEOREM 2.4. Let $y \in D$. Then the following are equivalent:

(a) $y \rightarrow y$ i.o. with respect to $\left\{Z_{n}\right\}$.

(b) $\sum_{n=1}^{\infty} \beta^{n}\left(y^{-1} N_{y}\right)=\infty$ for every open set $N_{y}$ containing $y$.

(c) $\sum_{n=1}^{\infty} \beta^{n}\left(N_{y}\right)=\infty$ for every open set $N_{y}$ containing $y$.

(d) $y \rightarrow y$ i.o. with respect to $\left\{W_{n}\right\}$.

(e) $\sum_{n=1}^{\infty} \beta^{n}\left(N_{y} y^{-1}\right)=\infty$ for every open set $N_{y}$ containing $y$.

Proof. We will prove that (a), (b) and (c) are equivalent. Then by dual arguments, (c), (d) and (e) also will be equivalent.

First, (a) implies (b), by the Borel-Cantelli Lemma.

Next, we show that (b) implies (c). We assume (b) and that there is an open set $N_{y}$ containing $y$ such that $\sum_{n=1}^{\infty} \beta^{n}\left(N_{y}\right)<\infty$. Let $N^{\prime}$ be a compact neighbourhood of $y$ such that $N^{\prime} \subset N_{y}$. Then $y^{-1} N^{\prime}$ is compact. Noting that for $x \in y^{-1} N^{\prime}, y x \in N^{\prime}$ and so we can find open sets $N_{x}(y)$ and $N(x)$ containing $y$ and $x$ respectively such that $N_{x}(y) N(x) \subset N_{y}$, we can find (using compactness) an open set $N$ containing $y$ such that $y^{-1} N \subset z^{-1} N_{y}$ for every $z$ in $N$. Now for every positive integer $k$,

$$
\sum_{n=1}^{\infty} \beta^{n}\left(N_{y}\right) \geqq \int\left[\sum_{n=k+1}^{\infty} \beta^{n-k}\left(z^{-1} N_{y}\right)\right] \beta^{k}(d z)
$$

which means that we can find a set $A$ such that, for every positive integer $k, \beta^{k}(A)=0$ and, for $z \notin A, \sum_{n=1}^{\infty} \beta^{n}\left(z^{-1} N_{y}\right)<\infty$. Hence there exists $w \in N$ such that $\sum_{n=1}^{\infty} \beta^{n}\left(w^{-1} N_{y}\right)<\infty$. Since $y^{-1} N \subset w^{-1} N_{y}$, this contradicts the assumption of (b).

Finally, we show that (c) implies (a). Let $k$ be an arbitrary positive integer and $N_{y}$ be an open set containing $y$. Then if (c) holds, we have $\sum_{j=1}^{k} \sum_{i=0}^{\infty} P\left(Z_{j+i k} \in N_{y}\right)=\infty$. So we can find an integer $m$ such that $1 \leqq m \leqq k$ and $\sum_{i=0}^{\infty} P\left(Z_{m+i k} \in N_{y}\right)=\infty$. Now

$$
\begin{aligned}
1 & \geqq P\left(Z_{n} \in N_{y} \text { finitely often }\right) \\
& \geqq \sum_{i=0}^{\infty} P\left(Z_{m+i k} \in N_{y}, Z_{n} \notin N_{y} \text { for all } n \geqq m+(i+1) k\right) \\
& \geqq \sum_{i=0}^{\infty} P\left(Z_{m+i k} \in N_{y}, Z_{m+i k}^{-1} Z_{n} \notin N_{y}^{-1} N_{y} \text { for all } n \geqq m+(i+1) k\right) \\
& =P\left(Z_{n} \notin N_{y}^{-1} N_{y} \text { for all } n \geqq k\right) \sum_{i=0}^{\infty} P\left(Z_{m+i k} \in N_{y}\right)
\end{aligned}
$$

which means that, for any open set $N_{y}$ containing $y$ and every positive 
integer $k$,

$$
P\left(Z_{n} \notin N_{y}^{-1} N_{y} \text { for all } n \geqq k\right)=0 .
$$

Now given an open set $N$ containing $y$, by Lemma 2.1, we can find an open set $N_{y}$ containing $y$ such that $N_{y}^{-1} N_{y} \subset y^{-1} N_{y}$. Then

$$
P_{y}\left(Z_{n} \in N \text { finitely often }\right) \leqq P\left(Z_{n} \in N_{y}^{-1} N_{y} \text { finitely often }\right)=0 .
$$

Hence $y \rightarrow y$ i.o. and (a) holds.

Theorem 2.5. Let $y \in D$ and $x \in K=$ the kernel of $D$. Then the following are equivalent:

(a) $x \rightarrow y$ i.o. with respect to $\left\{Z_{n}\right\}$.

(b) $\sum_{n=1}^{\infty} \beta^{n}\left(x^{-1} N_{y}\right)=\infty$ for every open set $N_{y}$ containing $y$.

(c) $x \rightarrow y$ and $\sum_{n=1}^{\infty} \beta^{n}\left(N_{y}\right)=\infty$ for every open set $N_{y}$ containing $y$.

Proof. First, (a) implies (b), by the Borel-Cantelli Lemma.

Next, we show that (b) implies (c). Suppose that (b) holds. Then by following the proof of "(c) implies (a)" in Theorem 2.4 and noting that

$$
\begin{array}{r}
P_{x}\left(Z_{m+i k} \in N_{y}, Z_{m+i k}^{-1} Z_{n} \notin N_{y}^{-1} N_{y} \text { for all } n \geqq m+(i+1) k\right) \\
=P_{x}\left(Z_{m+i k} \in N_{y}\right) \cdot P\left(Z_{n} \notin N_{y}^{-1} N_{y} \text { for all } n \geqq k\right),
\end{array}
$$

we see that, for every open set $N_{y}$ containing $y$ and each positive integer $k$, $P\left(Z_{n} \notin N_{y}^{-1} N_{y}\right.$ for all $\left.n \geqq k\right)=0$. Then it follows as in Theorem 2.4 that $y \rightarrow y$ i.o. This implies (c), by Theorem 2.4.

Finally, we show that (c) implies (a). Since (c) holds, by Theorem 2.4, $y \rightarrow y$ i.o. Since $x \rightarrow y, y \in x D$. But $x D$ is a minimal right ideal of $D, x$ being an element of $K$. Therefore, $x \in x D=y D$ so that $y \rightarrow x$. By Lemma 2.2, $x \rightarrow y$ i.o. The proof of the theorem is complete.

Remark. Using Theorem 2.4, it is now easy to show that the set $R$ of recurrent states of $\left\{Z_{n}\right\}$ is precisely the kernel $K$ of $D$. If, for each $y \in D, y$ is not recurrent, then by Theorem 2.4 we can find an open neighbourhood $N_{y}$ for each $y$ such that $\sum_{n=1}^{\infty} \beta^{n}\left(N_{y}\right)$ is finite. But then since $D$ is compact, $\sum_{n=1}^{\infty} \beta^{n}(D)$ is finite, which is absurd. Hence $R$ is nonempty. Also, $R$ is a left ideal of $D$. If $R^{\prime}$ is the set of recurrent states of the left random walk $\left\{W_{n}\right\}$, then, by Theorem $2.4, R=R^{\prime}$. Also, $R^{\prime}$ is a right ideal of $D$. Therefore, $R$ is a two-sided ideal of $D$ and hence contains $K$. By Lemma 2.3, $R \subset K$. Hence $R=K$. A direct proof of this interesting fact can also be given independently of Theorem 2.4. This is shown in what follows.

THEOREM 2.6. The set $R$ of recurrent states of $\left\{Z_{n}\right\}$ is precisely the kernel $K$ of $D$. 
Proof. Because of Lemma 2.3, suffice it to show that $K \subset R$. We will use the fact that for any regular probability measure $Q$ on $S$, the function $x \rightarrow Q\left(x^{-1} U\right)$ is lower semicontinuous for open $U$. Now let $x \in K$. For any $y \in x D$, we have $x \in y D$. For any open neighbourhood $U$ of $x, U \cap y D$ is nonempty. Thus for some $n, U \cap y F^{n}$ is nonempty. Let

$$
U_{n}=\left\{y \in x D: U \cap y F^{n} \text { is nonempty }\right\} .
$$

Then $U_{n}$ is relatively open in the compact set $x D$. So there is a finite subcover and, for some finite $n$,

$$
g(y) \equiv \sum_{j \leqq n} \beta^{j}\left(y^{-1} U\right)>0
$$

for all $y \in x D$. Then by the lower semicontinuity of $g(y)$, there is a $p>0$ such that $g(y) \geqq p$ for all $y \in x D$. Let $q=p / n$. For any positive integer $k$, the conditional probability that $x Z_{m} \notin U$ for $n k<m \leqq n(k+1)$, given any conditions on $X_{j}$ for $j \leqq n k$, is at most $1-q$. Hence

$$
P\left(x Z_{m} \notin U \text { for } n k<m \leqq n N\right) \leqq(1-q)^{N-k}
$$

which converges to zero as $N$ approaches infinity. This means that

$$
P_{x}\left(Z_{m} \in U \text { finitely often }\right)=0
$$

so that $x \in R$. The proof is complete.

ACKNOWLEDGEMENT. We are grateful to the referee for showing us the direct proof of Theorem 2.6.

ADDED IN PROOF. Theorem 2.5 is true for every $x, y \in D$, if $S$ is compact abelian. For inequality (1) (Proof of Lemma 2.2) (with $P_{x}, z, N_{z}$ replaced by $P, x, N_{x}$ resp.) shows that if $x \mapsto y$ i.o., then $P\left(Z_{n} \in N_{y}\right.$ f.o. $)>0$, a contradiction to a result of [10], since for every $y$ in the kernel $P\left[Z_{n} \in N_{y}\right.$ i.o. $]=1$ for every open set $N_{y}$ containing $y$.

\section{REFERENCES}

1. J. F. Berglund and K. H. Hofmann, Compact semitopological semigroups and weakly almost periodic functions, Lecture Notes in Math., no. 42, Springer-Verlag, New York, 1967. MR 36 \#6531.

2. K. L. Chung and W. J. Fuchs, On the distribution of values of sums of random variables, Mem. Amer. Math. Soc. No. 6 (1951), 1-12. MR 12, 722.

3. J. Larisse, Marches au hasard sur les demigroupes discrets: Classes essentielles, periodes, recurrence, C. R. Acad. Sci. Paris Sér. A-B 274 (1972), A339-A341.

4. - Marches au hasard sur les demigroupes discrets: Marches recurrentes et moyennes invariantes, C. R. Acad. Sci. Paris Sér. A-B 274 (1972), A413-A416.

5. R. M. Loynes, Products of independent random elements in a topological group, $Z$. Wahrscheinlichkeitstheorie und Verw. Gebiete 1 (1962/63), 446-455. MR 27 \#6293.

6. P. Martin-Löff, Probability theory on discrete semigroups, $\mathrm{Z}$. Wahrscheinlichkeitstheorie und Verw. Gebiete 4 (1965), 78-102. MR 32 \#1740. 
7. M. Rosenblatt, Limits of convolution sequences of measures, J. Math. Mech. 9 (1960), 293-305; Addendum, ibid. 10 (1961), 681. MR 22 \#2547; MR 22 \#9544.

8. - Stationary measures for random walks on semi-groups, Semigroups (Proc. Sympos. Wayne State Univ., Detroit, Mich., 1968), Academic Press, New York, 1969, pp. 209-220. MR 41 \#4666.

9. M. Rosenblatt, Markov processes: structure and asymptotic behavior, Grundlehren der math. Wissenschaften, Band 184, Springer-Verlag, New York, 1971.

10. T. C. Sun, A. Mukherjea and N. A. Tserpes, On recurrent random walks on semigroups, Trans. Amer. Math. Soc. (to appear).

Department of Mathematics, University of South Florida, Tampa, Florida 33620 (Current address of A. Mukherjea and N. A. Tserpes)

Department of Mathematics, Wayne State University, Detroit, Michigan 48202 (Current address of T. C. Sun) 